\title{
Re-Formulated Immuno-Editing as Agonist and Permissive Elements in Establishment of Foci of Carcinogenesis
}

\author{
Lawrence M Agius* \\ Department of Pathology, University of Malta Medical School, Malta
}

Submission: April 26, 2019; Published: May 28, 2019

"Correspondence Author: Lawrence M Agius, Department of Pathology, Mater Dei Hospital, Tal-Qroqq, University Of Malta Medical School, 27 Ballarat, Guzeppe Caruana Street, Tal-Virtu, Rabat, Rbt09 Malta, Europe

\begin{abstract}
Diffusional phenomena of paracrine and autocrine reconstitution in a focus of initial carcinogenesis include the derivative phenomenon of immuno-editing in the induced loss of immuno-surveillance. Immuno-surveillance is attempted definition of the constitutional identification of an injury that relates intimately with inflammatory response and interactivity. In terms further conforming to an injury in chronic inflammation, the responsive elements at reconstitution fail as a result of loss an antigenicity of individual tumor cells. Recapitulation of such agonist action is further promoted as permissive elemental injury that transforms such permissiveness to the emergence of tumor cells that evade the immune system. Characterization is injury-provoking within the systems of complex interactivity as modulated by multiple molecular events at attempted reconstitution.
\end{abstract}

\section{Introduction}

The concept of carcinogenesis is best accounted for by an inflammatory reactivity that includes many variably operative inducing agents, but which are mainly orchestrated by NFkappaB actions. Therapies targeting immune cell function have significant clinical efficacy in cancer [1]. In the ensuing development of a neoplastic lesion, it is instructive to consider the evolution of a neoplasm in terms of the associated growth factors, mitogens, chemokines and cytokines within a milieu that is itself responsive to immune mechanisms of operability. Tumors modulate the immune system by immune-editing the microenvironment, inducing cancer immune-surveillance, tumor dormancy and finally escape with ensuing tumor development [2]. Immuno-editing as a re-shaping of immunogenicity of tumors is an actual dysfunction of the relative dimensions for promotion and progression of malignant lesions that transcend the local focus for proliferative amplification within single and multiple organs of carcinogenesis. Anti-tumor immune responses place cancer cells under selective pressure to lose or down regulate target antigens, and vaccination against virus- or host-"driver" oncogenes are proposed to overcome immune escape [3].

\section{Promotional Modulation}

Dimensional recruitment of the various parameters of carcinogenesis account for immune modulation systems that involve further cooperative development of inflammatory reactivities. The immune microenvironment exerts a strong selection pressure in early-stage, untreated non-small-cell lung cancers that yields multiple routes to immune evasion [4]. According to the immune-editing theory, immune escape is essential for tumor survival, and this may yield a new basis for cancer treatment [5]. One needs to focus on the structure and dynamic functional organization of the entire immune system rather than on isolated individual components only [6]. In such manner, the implicit recruitment of bone-marrow stem cancer cells promotes an idealized immunity series of responses that both enhance and further propagate the institution of injurious agents in carcinogenesis. It is interesting, as well documented by the role of oncogenic viruses and bacteria, to consider the complex interactivities of agonist systems as system pathways of convergence and as systems of divergent reconstitution within the fundamental immune response. Regulatory lymphocytes play an important role in tumor evasion and infiltration of Treg cells in breast cancer lesions has been suggested as an independent unfavorable prognostic factor [7].

\section{Perpetuation of Injury}

The perpetuation of tissue injury within systems of pronounced reproducibility is the various dimensional reconstitutions of carcinogenesis that promote a reappraisal of the injury in terms paradoxically of the inflammatory responses, 
particularly in terms of a chronic inflammation that progresses beyond nuclear DNA injury. Hypoxia plays a critical role in cancer progression and in immune editing, which potentially results in solid tumors escaping immunosurveillance [8]. Also, metabolism of cancer cells and immune cells is effective in conditioning the tumor microenvironment [9]. It is further to the permissive relocation of the carcinogenic injury that the carcinomatous lesion both evolves and accounts for an extensive immuno-editing of the tumor antigenicity. Measurements of $\mathrm{T}$ cell infiltration in metastases by immunoscore allow some prognostic inflammation to be obtained to support immune editing by coevolving adapting immune responses [10]. It is in terms of permissive micro-environments that the neoplasm evades immune suppression within the variability response of tissue and cell injury. Epstein Barr virus infection determines the immune hallmarks of plasmablastic lymphoma and promotes a tolerogenic microenvironment [11]. Self-promoting de-evolution is a standard hallmark of a cancerous focus that indeed defines dynamics of responsiveness. Concurrent events overlap within the variability of response that includes dimensions of an either/ or phenomenon that operates as system reproducibility in the face of injury to multiple DNA sequences within the nucleus of the transforming cell or cells.

\section{Immuno-Editing}

The production of a lesion parameter that evokes immunoediting and immunosurveillance allows for a reconstitution of the injury in terms of basic parameters of permissive inflammatory response.

The definitive profile operability as distinguishable by pathway non-resolution is clearly an artifact lesion in terms of the permissive inflammatory status of a given initial focus of carcinogenesis. MHC-II presentation plays a central role in tumor evolution; the anti-cancer immune response against mutated peptides (neoantigens) is driven by MHC-I-restricted cytotoxic CD8+ T cell responses but MHC-II-restricted CD4+ T cells also drive anti-tumor responses [12]. It is in such manner that evolving support for loss of tumor antigenicity is itself an inherent attribute of carcinogenesis as this latter both promotes and permits progression within given template schemes for further reproducibility.

\section{Innate Immunity}

The innate immune system of response to a given focus of emerging carcinogenesis is a complex attribute within protean schemes of performance that call into operability the system interactions with a myriad of heterogeneous elements for further action. Chromosomal instability is a hallmark of human cancer and is a central driver of tumor evolution and a genomic source for the crosstalk with the tumour's microenvironment in the course of immune editing and evasion [13]. Agonists do not allow for re-characterization of the focus of carcinogenesis but instructs a reconstitution of permissive co-operability within an emerging neoplasm. It is highly likely that the focality of a carcinogenetic focus both promotes and re-establishes parameters for immuno-editing of the lesion beyond simple loss of antigenicity. It is significant to characterize parameters of an induced carcinogenesis pathway within the parametric dimensions of reproducible immuno-editing, as well illustrated by system profiles of permissiveness. There is significant spatial genomic heterogeneity even in the same prostate cancer patient, and this supports the origin of this neoplasm as either polyclonal or monoclonal, and hence with implications for treatment decisions [14].

\section{Co-Operability}

Depicted scenes for overlap co-operability are station phenomena in the reappraisal process for recognizable carcinogenesis. In such manner, the incremental proliferative response renders the dividing DNA strands as elements in reconstitution. In a manner that is both provocative and nonconstitutional, the neoplastic lesion is adaptive response to incremental change within antigen pathways of non-resolving systems of response. The immune system may select cancer cells that grow as these are fit to escape immune surveillance during tumor development [15]. The parameters for such response are permissive and agonistic within systems that otherwise reformulate the cooperative inducement for further loss of antigenicity, as assessed by mutant clones of dividing tumor cells. Immune editing shapes the cancer landscape [16].

\section{Interference Systems}

Permissive interference of subject proliferative dynamics is a constitutional system of dysregulation within pathways of conflicting modes of permissiveness in carcinogenesis. Losing the ability to present neoantigens through human leukocyte antigen loss may facilitate immune evasion in cancer [17]. It is significant to consider antigenicity as simple formula for interactivities of host cells subsequent to penetrant infectious agents such as Helicobacter pylori in gastric carcinogenesis and of Papillomavirus infection in cervical cancer. The importance for re-appraisal is further constitutive attribute for significant re-editing of antigens as well-documented by pathway molecular events in tumors. Immunotherapy has been presented as potential option for cancer treatment [18].

\section{Progression}

Attributes for further progression of a neoplastic focus is a borne-out phenomenon for the establishment of permissive loss of tumor cell antigenicity, as further promoted in systems of complex interactivity. T-lymphocyte-associated antigen 4, programmed cell death protein 1 , and programmed cell death protein ligand 1 are known as the "checkpoint inhibitors" and induce immunologic manipulation with remarkable antitumor efficacy [19]. The dimensions for recognition are documentary evidence for significant re-appraisal of a series of immune responses that further culminates in malignant transformation of host cells. The further cooperative dimensions include the 


\section{Cancer Therapy \& Oncology International Journal}

realization of injury as transformation. Such transformation includes the realization of significant complexity, a complexity that is carried through by dimensions of dysfunctional reproducibility.

\section{Concluding Remarks}

Immuno-surveillance and immuno-editing of tumor cell antigenicity are a basic structural re-constitution that best accounts for systems of response within spheres of operability and within attempts at constitutional correction. The emergence of pathway reproducibility allows for systems leading to amplification, as well illustrated by the phenomenon for reconstructive reformulation. The performance of attribute identification carries with it the dimensional structural anatomy of dysfunctional interactivity of carcinogenesis, as well formulated by dimensions of growth, recurrence and metastatic spread. It is further to be recognized the immediate inflammatory parameters of a phenomenon that is strictly amplifying rather than mutagenic. In terms of attempted reappraisal, the prospective emergence of a focus of carcinogenesis is defined reappraisal of antigenicity as borne out by inflammation and reconstitution.

\section{References}

1. LaFleur MW, Nguyen TH, Coxe MA, Yates KB, Trombley JD, et al. (2019) A CRISPR-Cas9 delivery system for in vivo screening of genes in the immune system. Nat Commun 10(1): 1668.

2. Bose D, Banerjee S, Chatterjee N, Das S, Saha M, et al. (2019) Inhibition of TGF-beta induced lipid droplets switches M2 macrophages to M1 phenotype. Toxic in Vitro 58: 207-214.

3. Dharmaraj N, Piotrowski SL, Huang C, Newton JM, Golfman LS, et al. (2019) Anti-tumor immunity induced by ectopic expression of viral antigens is transient and limited by immune escape. Oncoimmunology 8(4).

4. Rosenthal R, Cadieux EL, Salgado R, Bakir MA, Moore DA, et al. (2019) Neoantigen-directed immune escape in lung cancer evolution. Nature 567(7749): 479-485.

5. Duan S, Guo W, Xu Z, He Y, Liang C, et al. (2019) Natural killer group 2D receptor and its ligands in cancer immune escape Mol Cancer 18(1): 29.

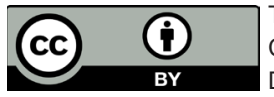

This work is licensed under Creative Commons Attribution 4.0 License

DOI: 10.19080/CTOIJ.2019.14.555877
6. Haas OA (2019) Primary immunodeficiency and cancer predisposition revisited: embedding two closely related concepts into an integrative conceptual framework. Front Immunol 9:3136.

7. Dziobek K, Biedka M, Nowikiewicz T, Szymankewicz M, Lukaszewska E, (2018) Analysis of Treg cell population in patients with breast cancer with respect to progesterone receptor status. Contemp Oncol (Pozn) 22(4): 236-239.

8. Ando Y, Siegler EL, Ta HP, Cinay GE, Zhou H, et al. (2019) Evaluating CAR-T cell therapy in a hypoxic 3D tumor model. Adv Health Mater $8(5)$

9. Li Y, Zhu B (2018) metabolism of cancer cells and immune cells in the tumor microenvironment. Front Immunol 9: 3080.

10. de Andrea CE, Schalper KA, Sanmamed MF, Melero I (2018) Immunodivergence in metastatic colorectal cancer. Cancer Cell 34(6): 876-878.

11. Gravelle P, Pericart S, Tosolini M, Fabiani B, Coppo P, et al. (2018) EBV infection determines the immune hallmarks of plasmablastic lymphoma. Oncoimmunology 7(10).

12. Marty Pyke R, Thompson WK, Salem RM, Font-Burgada J, Zanetti M, (2018) Evolutionary pressure against MHC Class II binding cancer mutations. Cell 175(2): 416-428.

13. Bakhoum SF, Cantley LC (2018) The multifaceted role of chromosomal instability in cancer and its microenvironment. Cell 174(6): 1347 1360 .

14. Su F, Zhang W, Zhang D, Zhang Y, Pang C, et al. (2018) Spatial intratumor genomic heterogeneity within localised prostate cancer revealed by single-nucleus sequencing. Eur Urol 74(5): 551-559.

15. Arrieta VA, Cacho-Diaz B, Zhao J, Rabadan R, Chen L (2018) The possibility of cancer immune editing in gliomas. A critical review. Oncoimmunology 7(7).

16. Starling S (2017) MHC molecules: immune editing shapes the cancer landscape. Nat Rev Immunol 17(12): 729.

17. McGranahan N, Rosenthal R, Hiley CT, Rowan AJ, Watkins TBK, et al (2017) Allele-specific HLA loss and immune escape in lung cancer evolution. Cell 171(6): 1259-1271.

18. Rusch T, Bayry J, Werner J, Shevchenko I, Bazhin AV (2018) Immunotherapy as an option for cancer treatment. Arch Immunol There Exp (Warsz) 66(2): 89-96.

19. Esin E (2017) Clinical applications in immunotherapy combination methods and new opportunities for the future. Biomed Res Int doi:10.1155.

\section{Your next submission with Juniper Publishers will reach you the below assets}

- Quality Editorial service

- Swift Peer Review

- Reprints availability

- E-prints Service

- Manuscript Podcast for convenient understanding

- Global attainment for your research

- Manuscript accessibility in different formats

( Pdf, E-pub, Full Text, Audio)

- Unceasing customer service

Track the below URL for one-step submission https://juniperpublishers.com/online-submission.php 